\title{
POSITIVITY CONDITIONS FOR HERMITIAN SYMMETRIC FUNCTIONS*
}

\author{
JOHN P. D'ANGELO ${ }^{\dagger}$ AND DROR VAROLIN ${ }^{\dagger}$
}

Dedicated to Yum-Tong Siu on the occasion of his sixtieth birthday

0. Introduction. We introduce a family of positivity conditions for Hermitian symmetric functions, establish basic properties, and connect the ideas with complex geometry. Let $M$ be a complex manifold, and let $M^{\prime}$ denote its complex conjugate manifold. In this paper $M$ will typically be either $\mathbf{C}^{n}$ or the total space of a holomorphic line bundle over a compact complex manifold.

A holomorphic function $R: M \times M^{\prime} \rightarrow \mathbf{C}$ is called Hermitian symmetric if

$$
R(z, \bar{w})=\overline{R(w, \bar{z})}
$$

for all $z$ and $w$ in $M$. Observe that $z \rightarrow R(z, \bar{z})$ is then necessarily a real-valued function; we say that $R$ is "real on the diagonal"; conversely, by polarization $R$ is determined by its values on the diagonal. Let $\mathcal{P}_{0}(M)$ denote the collection of Hermitian symmetric functions on $M \times M^{\prime}$. We will introduce in Definition 1 , for $N$ a positive integer or infinity, a subset $\mathcal{P}_{N}=\mathcal{P}_{N}(M)$ of $\mathcal{P}_{0}(M)$. On the diagonal, the set $\mathcal{P}_{1}$ consists by definition of those $R$ that are nonnegative, and the set $\mathcal{P}_{\infty}$ consists (by a classical result recalled in Lemma 2) of squared norms of Hilbert space valued holomorphic mappings. The subsets $\mathcal{P}_{k}$ therefore interpolate two natural but distinct notions of nonnegativity for Hermitian symmetric functions. The condition defining $\mathcal{P}_{k}$ is analogous to a positivity property for higher curvatures of Hermitian metrics.

We show, as part of Theorem 3 , that $\mathcal{P}_{j}\left(\mathbf{C}^{2}\right) \neq \mathcal{P}_{k}\left(\mathbf{C}^{2}\right)$ when $j \neq k$. Theorem 3 includes precise information about when a member of a discrete collection of natural one-parameter families of Hermitian symmetric polynomials lies in $\mathcal{P}_{k}\left(\mathbf{C}^{2}\right)$.

Our other main result (Theorem 1) concerns a stability criterion. A subset $S$ of $\mathcal{P}_{0}(M)$ is called stable if there is a finite $k$ for which $\mathcal{P}_{k} \cap S=\mathcal{P}_{\infty} \cap S$. The minimum such $k$ is called the stability index of $S$, and is written $I(S)$. For example, it is standard that the collection $H$ of nonnegative Hermitian forms on $\mathbf{C}^{n}$ equals the collection of squared norms of linear forms; therefore $I(H)=1$. For each integer $N$ we give an example of an $S$ for which $I(S)=N$. In Theorem 1 we relate the stability index to the number of positive eigenvalues of the underlying matrix of coefficients of a Hermitian symmetric function.

The concepts in this paper apply in a situation of some interest in complex geometry. Let $X$ be a complex manifold and let $L$ be a holomorphic line bundle over $X$. A (possibly degenerate) metric $g$ on the fibres of $L$ is called a globalizable singular metric if it is the restriction of a Hermitian symmetric function $G$ on $L \times L^{\prime}$. Our focus here will be on $G$. The subsets $\mathcal{P}_{N}$ of these metrics provide intermediate conditions between simply being a (possibly degenerate) metric $\left(\mathcal{P}_{1}\right)$ and being a holomorphic pullback of the Fubini-Study metric $\left(\mathcal{P}_{\infty}\right)$.

*Received June 7, 2003; accepted for publication August 29, 2003.

†Department of Mathematics, University of Illinois at Urbana-Champaign, Urbana, IL 61801, USA (jpda@math.uiuc.edu; dror@math.uiuc.edu). 
The proof of Theorem 1 applies to show that the stability index for the collection $S$ of globalizable metrics on a holomorphic line bundle $L$ over a compact complex manifold $X$ is bounded above by $\operatorname{dim} H^{0}\left(X, L^{*}\right)-1$. Thus $S$ is stable. See Theorem 2 .

There are many other useful positivity conditions for Hermitian symmetric functions. In particular, the set of nonnegative logarithmically plurisubharmonic (Hermitian) functions properly contains $\mathcal{P}_{2}$. We briefly discuss these issues in Section V.

The authors wish to acknowledge useful discussions over the years with Yum-Tong $\mathrm{Siu}$; in particular Siu pointed out to the first author a connection between positivity conditions for bihomogeneous polynomials and isometric imbedding. Motivated by Siu's comments, the first author and Catlin [CD2] proved an isometric imbedding theorem for holomorphic vector bundles. This theorem then motivated much of the work in the current paper. The authors also wish to thank Dan Lichtblau of Wolfram Research, who did a Groebner basis computation which provided useful insight, and Dan Grayson for some useful discussions.

The first author acknowledges support from NSF grant DMS-0200551.

1. Positivity Classes for Hermitian symmetric functions. We begin by describing an essentially general class of Hermitian symmetric functions on a complex manifold $M$. Let $\mathcal{H}$ be a Hilbert space with inner product $\langle$,$\rangle . Let f, g: M \rightarrow \mathcal{H}$ be holomorphic mappings, and define $R$ by the formula

$$
R(z, \bar{w})=\langle f(z), f(w)\rangle-\langle g(z), g(w)\rangle .
$$

Then $R$ is Hermitian symmetric. When $g=0$ in (1) we have $R=\|f\|^{2}$ on the diagonal; squared norms of holomorphic mappings will play a special role in this paper. We also note that, by choosing $\mathcal{H}=\mathbf{C}$, and by choosing $f$ and $g$ appropriately in (1), we can obtain the (polarization of the) real and imaginary parts of an arbitrary holomorphic complex-valued function.

When $R$ satisfies (1), we use the term holomorphic representation to denote the holomorphic mapping $(f, g): M \rightarrow \mathcal{H} \times \mathcal{H}$ determining $R$. When $g$ can be chosen to be 0 , we simply say that $f: M \rightarrow \mathcal{H}$ is a holomorphic mapping representing $R$.

We now introduce the positivity classes $\mathcal{P}_{k}(M)$ for the collection of Hermitian symmetric functions on the manifold $M \times M^{\prime}$. Our notion evokes some classical functional analysis such as Bochner's theorem on functions of positive type; the key difference is that we focus on nonnegativity of matrices of a fixed size rather than of all sizes.

Definition 1. (Positivity classes). Let $M$ be a complex manifold. We denote the set of Hermitian symmetric function on $M \times M^{\prime}$ by $\mathcal{P}_{0}(M)$. For each positive integer $N$ we write $R \in \mathcal{P}_{N}(M)$ if

$$
\sum_{i, j=1}^{N} R\left(z_{i}, \bar{z}_{j}\right) a_{i} \bar{a}_{j} \geq 0
$$

for all $z=\left(z_{1}, \ldots, z_{N}\right) \in M^{N}$ and all $a \in \mathbf{C}^{N}$. In other words the Hermitian matrix with $(i, j)$ entry equal to $R\left(z_{i}, \bar{z}_{j}\right)$ is nonnegative definite. We write $R \in \mathcal{P}_{\infty}$ when $R \in \mathcal{P}_{N}$ for all $N$; thus 


$$
\mathcal{P}_{\infty}=\bigcap_{N=1}^{\infty} \mathcal{P}_{N}
$$

When $M$ is fixed, we drop it from the notation, and write $\mathcal{P}_{N}$ for $\mathcal{P}_{N}(M)$.

REMARK 1. For each subset $\mathcal{P}_{N}$ there are corresponding sharp forms; for example we could demand that the matrix $R\left(z_{i}, \bar{z}_{j}\right)$ be positive definite whenever the points are distinct. In some contexts other sharp forms are useful; see Definition 5 in Section V.

Definition 2. (Stability Index) Let $S$ be a subset of $\mathcal{P}_{0}$. We define $I(S)$ to be the smallest $k$ for which

$$
S \cap \mathcal{P}_{\infty}=S \cap \mathcal{P}_{k}
$$

If no such $k$ exists we write $I(S)=\infty$. When $I(S)$ is finite we say that $S$ is stable. $\mathcal{P}_{\infty}$

We begin by noting some obvious properties of the sets $\mathcal{P}_{k}$, and then we study

Lemma 1. Each $\mathcal{P}_{k}$ is closed under sum and under product. For each $k$ we have $\mathcal{P}_{k+1} \subset \mathcal{P}_{k}$. If $R_{\lambda}$ is a family of Hermitian symmetric functions depending continuously on some parameter $\lambda$, then the set of $\lambda$ for which $R_{\lambda} \in \mathcal{P}_{k}$ is closed.

Proof. These facts follow easily from Definition 1. We note that the proof of closure under product uses a well-known lemma of Schur: if $\left(a_{i j}\right)$ and $\left(b_{i j}\right)$ are nonnegative definite matrices of the same size, then their Schur product $\left(a_{i j} b_{i j}\right)$ is also nonnegative definite. See $[\mathrm{AM}]$ or $[\mathrm{D} 4]$.

Lemma 2. Suppose that there is a Hilbert space $\mathcal{H}$ and a holomorphic function $f: M \rightarrow \mathcal{H}$ such that

$$
R(z, \bar{w})=\langle f(z), f(w)\rangle .
$$

Then $R \in \mathcal{P}_{\infty}$. Conversely, if $R \in \mathcal{P}_{\infty}$, then there is a Hilbert space $\mathcal{H}$ and a holomorphic mapping $f: M \rightarrow \mathcal{H}$ representing $R$, and thus (2) holds.

Proof. First assume (2) holds. Fix $N$, and choose arbitrary points $z_{i}$ in $M$ and $a \in \mathbf{C}^{N}$. We see that

$$
\sum_{i, j=1}^{N} R\left(z_{i}, \bar{z}_{j}\right) a_{i} \bar{a}_{j}=\sum_{i, j=1}^{N}\left\langle f\left(z_{i}\right) a_{i}, f\left(z_{j}\right) a_{j}\right\rangle=\left\|\sum_{i=1}^{N} f\left(z_{i}\right) a_{i}\right\|^{2} \geq 0
$$

and hence $R \in \mathcal{P}_{N}$ for all $N$.

The converse assertion is classical; various versions go back to Mercer in 1909 and to E. H. Moore in 1916. For historical remarks and related ideas we refer to [A], $[\mathrm{AM}]$, and $[\mathrm{S}]$.

We give a sketch, following [CW], of the converse assertion. Consider the complex vector space $V$ of functions on $M$ with finite support. Using $R$ we define a Hermitian form on $V$ by the following formula. For $u, v \in V$ we put

$$
\langle u, v\rangle_{R}=\sum R(z, \bar{w}) u(z) \overline{v(w)},
$$


where the sum is taken over all $z, w \in M$, but is finite by the support condition. Let $V_{0}$ denote the collection of $u$ with $\langle u, u\rangle_{R}=0$. It follows from the nonnegative definiteness of all matrices $R\left(z_{i}, \bar{z}_{j}\right)$ that $V_{0}$ is a subspace of $V$. The quotient space is then an inner product space under $\langle,\rangle_{R}$, and we take $\mathcal{H}$ to be its completion. For $z \in M$ we define $f(z)$ to be the image under the quotient map of the function with support at the single point $z$ and value unity there. Since $R$ is holomorphic on $M \times M^{\prime}$, it follows that $f: M \rightarrow \mathcal{H}$ is holomorphic.

Next we give a simple example showing that $\mathcal{P}_{1}(M)$ and $\mathcal{P}_{2}(M)$ are distinct sets even in very simple situations. This example also gives insight into the stability index.

ExAmple 1. Let $M=\mathbf{C}^{2}$. For each real number $c$ we define $R_{c}$ by

$$
R_{c}(z, \bar{w})=z_{1}^{2} \bar{w}_{1}^{2}+(c-2) z_{1} z_{2} \bar{w}_{1} \bar{w}_{2}+z_{2}^{2} \bar{w}_{2}^{2}
$$

It is elementary to check that $R_{c} \in \mathcal{P}_{1}$ if and only if $c \geq 0$ whereas $R_{c} \in \mathcal{P}_{2}$ if and only if $c \geq 2$. In fact, for each $k$ larger than $2, R_{c} \in \mathcal{P}_{k}$ if and only if $c \geq 2$; therefore, if $S=\left\{R_{c}: c \geq 0\right\}$, then $I(S)=2$. Recall by contrast that $I(H)=1$ when $H$ is the set of nonnegative Hermitian forms.

We recall the standard test for nonnegative definiteness of an $N$ by $N$ matrix and provide the appropriate caution. A Hermitian matrix is nonnegative definite if and only if every principal minor determinant is nonnegative. It is not sufficient to assume only that every leading principal minor determinant is nonnegative. The matrix

$$
\left(\begin{array}{ccc}
1 & 0 & 0 \\
0 & 0 & 0 \\
0 & 0 & -1
\end{array}\right)
$$

has leading principal minor determinants of 1,0 , and 0 , and yet it has a negative eigenvalue. See [D4] for more discussion.

We immediately obtain a method for deciding whether $R \in \mathcal{P}_{k}$. Given $k$ points $z_{1}, \ldots, z_{k}$ in $M$, we put $\Delta_{k}^{R}(z)=\operatorname{det}\left(R\left(z_{i}, \bar{z}_{j}\right)\right)$.

Lemma 3. Suppose $R \in \mathcal{P}_{0}(M)$ and $k \geq 1$. Then $R \in \mathcal{P}_{k}$ if and only if $R \in \mathcal{P}_{k-1}$ and $\Delta_{k}^{R}(z)=\operatorname{det}\left(R\left(z_{i}, \bar{z}_{j}\right)\right) \geq 0$ for all $z=\left(z_{1}, \ldots, z_{k}\right) \in M^{k}$.

Next we give a method for computing $\Delta_{k}^{R}(z)$ in many cases. We say that $R$ has finite rank if there is a finite-dimensional Hilbert space representation for $R$; in other words, if there are holomorphic mappings $f, g: M \rightarrow \mathbf{C}^{K}$ such that

$$
R(z, \bar{w})=\langle f(z), f(w)\rangle-\langle g(z), g(w)\rangle
$$

For example, every polynomial function $R$ has finite rank. See [D4] for a simple proof. One notes that globalizable metrics (See Definition 3) on a holomorphic line bundle $L$ over a compact complex manifold also have finite rank, simply because the space of global sections of $L^{*}$ is finite-dimensional.

Suppose that $R$ has finite rank. For each $z=\left(z_{1}, \ldots, z_{k}\right) \in M^{k}$ we consider a $k$ by $k$ matrix whose $i, j$ entry is $h_{j}\left(z_{i}\right)$, where $h_{j}$ is a component function of either $f$ or $g$. We let $\Delta_{k, m}^{R}(z)$ denote the tuple of determinants of all such matrices, excluding the obvious repetitions, for which precisely $m$ of the column vectors are components of $g$. We write $\left\|\Delta_{k, m}^{R}(z)\right\|^{2}$ for the sum of squared absolute values of all these determinants.

We have the formula 


$$
\operatorname{det}\left(R\left(z_{i}, \bar{z}_{j}\right)\right)=\Delta_{k}^{R}(z)=\sum_{m=0}^{k}(-1)^{m}\left\|\Delta_{k, m}^{R}(z)\right\|^{2} .
$$

Formula (3) expresses the determinant $\Delta_{k}^{R}(z)$ in terms of the components of $f$ and $g$ in a somewhat tractable fashion. The proof is an elementary computation in exterior algebra. One computes the wedge product of the column vectors of the matrix $\operatorname{det}\left(R\left(z_{i}, \bar{z}_{j}\right)\right)$, expands by the distributive law, and uses the formula for the determinant of a $k$ by $k$ matrix in terms of $k$-th exterior powers. Formula (3) results. We note also that formula (29) from Section $V$ provides a proof and additional insight when $k=2$.

Formula (3) immediately combines with Lemma 3 to yield a necessary and sufficient condition for an $R$ with finite rank to lie in some $\mathcal{P}_{k}$ :

Proposition 1. $R \in \mathcal{P}_{k}$ if and only if $\sum_{m=0}^{j}(-1)^{m}\left\|\Delta_{j, m}^{R}(z)\right\|^{2} \geq 0$ for $1 \leq j \leq k$.

We now turn to our first main result. Theorem 1 generalizes the simple fact that, for scalar-valued entire holomorphic functions $f$ and $g$ on $\mathbf{C}^{n}$, the function $|f|^{2}-|g|^{2}$ can be nonnegative only when $g$ is a constant multiple of $f$. For general $k$, if $R=\|f\|^{2}-|g|^{2} \in \mathcal{P}_{k}$ and $f$ has too few linearly independent components, then $g$ must be a linear combination of the components of $f$, and thus $R$ actually must be a squared norm.

Let $R$ be a Hermitian symmetric function on $\mathbf{C}^{n}$ with Taylor expansion

$$
R(z, \bar{z})=\sum_{a, b} c_{a b} z^{a} \bar{z}^{b}
$$

We let $N_{+}(R)$ and $N_{-}(R)$ denote the number of positive and negative eigenvalues of the (perhaps infinite) Hermitian matrix $c_{a b}$. We allow the value infinity. In Theorem 1 we will not assume that $R$ has finite rank, although the situation reduces easily to that case.

Theorem 1. Let $R$ be a Hermitian symmetric function on $\mathbf{C}^{n}$ such that $R \in \mathcal{P}_{k}$. Then either $N_{-}(R)=0$ or $N_{+}(R) \geq k+1$.

Proof. We will work solely on the diagonal. If $N_{+}(R) \geq k+1$, then we are done. Suppose otherwise that $R \in \mathcal{P}_{k}, N_{-}(R)>0$, and $N_{+}(R) \leq k$. Since $N_{+}(R)$ is finite, we may write $R=\|f\|^{2}-\|g\|^{2}$ as usual. In view of Lemma 1, by adding an appropriate squared norm to $R$, we may suppose the following hold: $g$ takes values in $\mathbf{C}, N_{-}(R)=1, f$ takes values in $\mathbf{C}^{k}$, and that $N_{+}(R)=k$.

Choose $k$ points $z_{1}, \ldots, z_{k} \in \mathbf{C}^{n}$. We define the square matrix $A(f)(z)$ and column vector $G(z)$ by

$$
A(f)(z)=\left(\begin{array}{cccc}
f_{1}\left(z_{1}\right) & f_{2}\left(z_{1}\right) & \ldots & f_{k}\left(z_{1}\right) \\
f_{1}\left(z_{2}\right) & f_{2}\left(z_{2}\right) & \ldots & f_{k}\left(z_{2}\right) \\
\ldots & \ldots & & \ldots \\
f_{1}\left(z_{k}\right) & f_{2}\left(z_{k}\right) & \ldots & f_{k}\left(z_{k}\right)
\end{array}\right) \text { and } G(z)=\left(\begin{array}{c}
g\left(z_{1}\right) \\
g\left(z_{2}\right) \\
\ldots \\
g\left(z_{k}\right)
\end{array}\right)
$$

Consider the system of $k$ linear equations $A(f)(z) c(z)=G(z)$ for $k$ unknowns $c_{j}(z)$. Since the functions $f_{i}$ are linearly independent, the generic value of $\operatorname{det}(A(f)(z)$ 
is nonzero. The solution to the system is therefore given as the ratio of two determinants by Cramer's rule. We let $B_{j}(f, g)(z)$ denote the matrix obtained by replacing the $j$-th column in $A(f)(z)$ with $G(z)$. Thus

$$
c_{j}(z)=\frac{\operatorname{det}\left(B_{j}(f, g)(z)\right)}{\operatorname{det}(A(f)(z))} .
$$

On the other hand, when $g$ is scalar-valued, (3) simplifies to give:

$$
\operatorname{det}\left(R\left(z_{i}, \bar{z}_{j}\right)\right)=\Delta_{k}^{R}(z)=|\operatorname{det}(A(f)(z))|^{2}-\sum_{j=1}^{k}\left|\operatorname{det}\left(B_{j}(f, g)(z)\right)\right|^{2}
$$

Since $R \in \mathcal{P}_{k}$, the determinant on the left-hand side of (5) is nonnegative. Each term in the sum on the right-hand side of (5) is therefore bounded by $|\operatorname{det} A(f)(z)|^{2}$. Combining this fact with (4) shows that each $c_{j}(z)$ is a bounded meromorphic function, and hence a constant, written $c_{j}$. We therefore obtain, for each $i$,

$$
g\left(z_{i}\right)=\sum_{j} c_{j} f_{j}\left(z_{i}\right)
$$

Since the points $z_{i}$ are arbitrary, $g$ is a linear combination of the $f_{j}$; this statement contradicts the original assertion that $N_{-}(R) \neq 0$.

Corollary 1. Let $S_{k}$ denote the collection of Hermitian symmetric functions on $\mathbf{C}^{n}$ whose underlying matrices of Taylor coefficients have at most $k$ positive eigenvalues. Then $I\left(S_{k}\right) \leq k$ and $S_{k}$ is stable.

The proof of Theorem 1 yields a test for whether $g$ is a linear combination of the $f_{j}$. See Proposition 3 and Theorem 2 for an alternative point of view.

The proof of Theorem 1 goes through with essentially no change if $\mathbf{C}^{n}$ is replaced with a holomorphic vector bundle over a compact manifold, or, more generally, with a complex manifold admitting no nonconstant bounded holomorphic functions. On the other hand, no result resembling Theorem 1 holds for Hermitian symmetric functions on bounded domains in $\mathbf{C}^{n}$. It is easy to write down, for an arbitrary $j$, a Hermitian symmetric function $R$ on the unit ball for which $N_{+}(R)=1$ and $N_{-}(R)=j$.

2. Globalizable metrics. Hermitian symmetric functions arise naturally in complex geometry. In several important contexts Hermitian symmetric functions (nonnegative on the diagonal) restrict to (possibly singular) Hermitian metrics, and have played a key role in analytic geometry. See [Siu] and [CD2].

Let $X$ be a compact, complex manifold, and suppose that $L$ is a holomorphic line bundle over $X$. The complex vector space $H^{0}\left(X, L^{*}\right)$ of sections of the dual bundle $L^{*}$ is finite-dimensional. Let $C$ be a Hermitian form on $H^{0}\left(X, L^{*}\right)$. We associate with $C$ the Hermitian symmetric function $R_{C}$ on $L \times L^{\prime}$ as follows. If $\left\{\phi_{\alpha}\right\}$ is a basis for $H^{0}\left(X, L^{*}\right)$, and $c_{\alpha \beta}=\left\langle C \phi_{\alpha}, \phi_{\beta}\right\rangle$, then

$$
R_{C}(z, \bar{w})=\sum_{\alpha, \beta} c_{\alpha \beta} \phi_{\alpha}(z) \bar{\phi}_{\beta}(w) .
$$

Definition 3. Let $C$ be a Hermitian form on $H^{0}\left(X, L^{*}\right)$. The function $R_{C}$ defined on $L \times L^{\prime}$ by (6) is called a globalizable singular metric on $L$. 
Suppose that $C$ is positive semi-definite; then, for each $\phi \in H^{0}\left(X, L^{*}\right)$, the function $R_{C}$ is nonnegative on the diagonal. On the other hand, simple examples (see Example 1 and Theorem 3) show that the function defined by (6) can be nonnegative on the diagonal even when $C$ has some negative eigenvalues. In case $C$ has negative eigenvalues the mapping $g$ in a holomorphic representation of $R_{C}$ must be nonzero. Hence the intermediate positivity classes $\mathcal{P}_{k}$ provide useful geometric information.

A globalizable singular metric $G$ on $L$ can be written

$$
G=\sum_{\alpha, \beta} c_{\alpha \beta} \phi_{\alpha} \bar{\phi}_{\beta},
$$

where $\left\{\phi_{\alpha}\right\}$ form a basis for $H^{0}\left(X, L^{*}\right)$. The (necessarily) Hermitian matrix $\left(c_{\alpha \beta}\right)$ is called the underlying matrix of $G$.

We will drop the adjective singular and refer to $G$ as a globalizable metric on $L$. We use the term metric because $G$ restricts to a Hermitian metric on the fibres of $L$. In other words, there is a (possibly singular) metric $g$ in the usual sense for which $G(z, \bar{w})=g(z, \bar{w})$ whenever $\pi(z)=\pi(w)$. Here $\pi: L \rightarrow X$ is the usual projection. In [CD2] the metric $g$ is defined to be globalizable when a $G$ extending $g$ and satisfying (7) exists.

Complex projective space $\mathbf{P}_{n-1}$ and powers of the universal bundle $\mathbf{U}^{m}$ provide nice examples. The usual Fubini-Study metric $G$ on $\mathbf{U}$ is globalizable.

The dual bundle $\mathbf{H}^{m}$ of $\mathbf{U}^{m}$ is the $m$-th power of the hyperplane bundle; it is generated by global sections which we may identify with homogeneous polynomials of degree $m$. It is natural to equip $\mathbf{U}^{m}$ with the Hermitian metric $G^{m}$ given by the $m$-th tensor power of the Fubini-Study metric on $\mathbf{U}$. The metric $G^{m}$ provides another simple example of a globalizable metric. In the particular case of $\mathbf{U}^{m}$, a globalizable metric may be identified with a bihomogeneous polynomial on $\mathbf{C}^{n}$ :

$$
R(z, \bar{w})=\sum_{|\alpha|=m,|\beta|=m} c_{\alpha \beta} z^{\alpha} \bar{w}^{\beta} .
$$

The matrix of coefficients $\left(c_{\alpha \beta}\right)$ is Hermitian symmetric and $R(z, \bar{z})$ nonnegative. For $\mathbf{U}^{m}$, the natural metric $G^{m}$ can be identified with the bihomogeneous polynomial defined by

$$
R(z, \bar{w})=\langle z, w\rangle^{m} .
$$

In Theorem 3 we will consider perturbations of this particular $R$.

We interpret one piece of Lemma 1 in this setting.

Corollary 2. Suppose that $(L, R)$ and $(E, G)$ are holomorphic line bundles over a complex manifold $X$ with the indicated globalizable metrics $R$ and $G$. Suppose that $R$ and $G$ are in $\mathcal{P}_{k}$. Then $R \otimes G \in \mathcal{P}_{k}$.

Proof. The result follows from Lemma 1 , because $\mathcal{P}_{k}$ is closed under product.

Corollary 2 implies that the natural tensor product metric on a power of a line bundle lies in the same class $\mathcal{P}_{k}$ as does the original metric. When $k=2$ the converse of this fact plays a crucial role in the proof of the isometric imbedding theorem in [CD2]. 
3. Bihomogeneous polynomials, metrics, and the functions $\Sigma^{k}$. In this section we give an alternative manner for verifying that $R \in \mathcal{P}_{k}$ when $R$ is a bihomogeneous polynomial, or more generally, a globalizable metric. We begin with a general lemma.

Lemma 4. Let $M$ be a complex manifold and $\mathcal{H}$ be a Hilbert space with inner product $\langle$,$\rangle . Suppose that f, g: M \rightarrow \mathcal{H} \times \mathcal{H}$ is a Hilbert space representation of the Hermitian symmetric function $R$. Then $R \in \mathcal{P}_{N}$ if and only if

$$
\left\|\sum_{i=1}^{N} f\left(z_{i}\right) a_{i}\right\|^{2} \geq\left\|\sum_{i=1}^{N} g\left(z_{i}\right) a_{i}\right\|^{2}
$$

for all choices of $N$ points $z_{i} \in M$ and all $a \in \mathbf{C}^{N}$.

Proof. The computation is virtually the same as the proof of Lemma 2, and hence is left to the reader.

We recall that a bihomogeneous polynomial $R$ on $\mathbf{C}^{n} \times\left(\mathbf{C}^{n}\right)^{\prime}$ is a polynomial in $z$ and $\bar{w}$ that is homogeneous of the same degree in both sets of variables. Equivalently, for $\lambda \in \mathbf{C}$,

$$
R(\lambda z, \overline{\lambda z})=|\lambda|^{2 m} R(z, \bar{z})
$$

A bihomogeneous polynomial is real on the diagonal if and only if it is Hermitian symmetric, and this symmetry holds if and only if the matrix of coefficients of $R$ is Hermitian symmetric. See [D2] and [D4] for discussion of bihomogeneous polynomials.

We may identify a Hermitian symmetric nonnegative bihomogeneous polynomial $R$ of degree $2 m$ on $\mathbf{C}^{n}$ with a globalizable metric on $\mathbf{U}^{m}$ over $\mathbf{P}_{n-1}$. To see this fact, we write $R$, as in the definition of globalizable metric, in the form (7), where $\phi_{\alpha}$ is the monomial $z^{\alpha}$. The dual bundle $\mathbf{H}^{m}$ is generated by global sections which we may identify with homogeneous polynomials of degree $m$.

Let now $X$ be a compact complex manifold and $\pi: L \rightarrow X$ a holomorphic line bundle. We denote the pairing of a section $s$ of $L^{*}$ with a vector $v \in L$ by $s[v]$. We obtain Hermitian symmetric functions $R: L \times L^{\prime} \rightarrow \mathbf{C}$ by mimicking the situation for bihomogeneous polynomials.

Every Hermitian symmetric function $R$ on $L \times L^{\prime}$ is given, after diagonalizing the associated Hermitian form $C$ from (6) by the formula

$$
R(v, \bar{w})=\sum_{j=1}^{m} \mu_{j} s_{j}(\pi(v))[v] \overline{s_{j}(\pi(w))[w]} .
$$

In (10) the $\mu_{j}$ are the nonzero eigenvalues of $C$ and $s_{1}, \ldots, s_{k}$ are linearly independent elements of $H^{0}\left(X, L^{*}\right)$. By collecting the terms according to the sign of $\mu_{j}$ we write $R=\|f\|^{2}-\|g\|^{2}$, where the components of $f$ and $g$ are global sections of $L^{*}$. Thus the sections determine a (finite-dimensional) Hilbert space representation for $R$, and $R \in$ $\mathcal{P}_{1}$ precisely when $R$ is a globalizable metric. The following simple result characterizes when $R \in \mathcal{P}_{k}$.

Proposition 2. Let $\pi: L \rightarrow X$ be a holomorphic line bundle over a compact complex manifold $X$. Let $R$ be the Hermitian symmetric function defined on the diagonal by $R=\|f\|^{2}-\|g\|^{2}$. Then $R \in \mathcal{P}_{k}$ if and only if 


$$
\left\|\sum_{i=1}^{k} f\left(w_{i}\right)\right\|^{2} \geq\left\|\sum_{i=1}^{k} g\left(w_{i}\right)\right\|^{2}
$$

for all $w_{1}, \ldots, w_{k}$ in $X$.

Proof. Let $s$ be a section of $L^{*}$, and choose $v \in L$. For $a \in \mathbf{C}$ and $p \in X$, we have $s(p)[a v]=s(p)[w]$ for some $w \in L$. The proposition follows by combining this fact with formula (8) and the conclusion of Lemma 2.

We introduce notation for the functions appearing inside the norms in (11).

Definition 4. Let $X$ be a set, $G$ be an additive Abelian group, and $f: X \rightarrow G$ a function. For each positive integer $N$ we define $\Sigma^{N} f: X^{N} \rightarrow G$ by

$$
\left(\Sigma^{N} f\right)\left(z_{1}, \ldots, z_{N}\right)=\Sigma_{i=1}^{N} f\left(z_{i}\right) .
$$

We can then interpret the conclusion of Proposition 2 in several ways.

Corollary 3. Let $R$ be a Hermitian symmetric function on $L \times L^{\prime}$, written in the form $R=\|f\|^{2}-\|g\|^{2}$ as in Proposition 2. Then $R \in \mathcal{P}_{N}$ if and only if $\left\|\Sigma^{N} f\right\|^{2} \geq\left\|\Sigma^{N} g\right\|^{2}$.

Corollary 4 . Let $R_{\lambda}$ be defined on the diagonal of $L \times L^{\prime}$ by $R_{\lambda}=\|f\|^{2}-\lambda\|g\|^{2}$. Let $S=\left\{R_{\lambda}\right\}$. If there is an $N$ such that the variety $V\left(\Sigma^{N} f\right)$ is not contained in the variety $V\left(\Sigma^{N} g\right)$, then $I(S) \leq N$.

Let $X$ be a compact complex manifold, and let $L \rightarrow X$ be a holomorphic line bundle. We let $h=\operatorname{dim} H^{0}\left(X, L^{*}\right)$, By Definition $4, \Sigma^{k} s: L^{k} \rightarrow \mathbf{C}$ is defined by

$$
\left(\Sigma^{k} s\right)\left(v_{1}, \ldots, v_{k}\right)=\sum_{j=1}^{k} s\left[v_{j}\right],
$$

and is a section of the vector bundle $L^{k} \rightarrow X^{k}$. We will consider the zero varieties

$$
V\left(\Sigma^{k} s\right):=\left\{\left(x_{1}, \ldots, x_{k}\right) \in X^{k} ; \Sigma^{k} s \mid L_{x_{1}} \times \ldots \times L_{x_{k}} \equiv 0\right\}
$$

Proposition 3. Let $g, s_{1}, \ldots, s_{N} \in H^{0}\left(X, L^{*}\right)$, and suppose that

$$
\bigcap_{j=1}^{N} V\left(\Sigma^{h} s_{j}\right) \subset V\left(\Sigma^{h} g\right) .
$$

Then $g$ is a linear combination of $s_{1}, \ldots, s_{N}$.

Lemma 5. Let $T \in H^{0}\left(X, L^{*}\right)^{*}$. Then there exist points $v_{1}, \ldots, v_{h} \in L$ such that for all $s \in H^{0}\left(X, L^{*}\right)$,

$$
T s=\Sigma^{h} s\left(v_{1}, \ldots, v_{h}\right) .
$$

Proof. We may assume $T \neq 0$. Choose a basis $s^{1}, \ldots, s^{h}$ of $H^{0}\left(X, L^{*}\right)$ such that

$$
T s^{1}=1 \quad \text { and } \quad T s^{j}=0 \text { for } j=2, \ldots, h .
$$


Select vectors $u_{1}, \ldots, u_{h}$ such that the matrix with components $A_{j}^{i}:=s^{i}\left(u_{j}\right)$ is invertible. This choice is trivial (as is the Lemma) when $h=1$. For $h \geq 2$ selecting such vectors is also possible; for instance, we may choose

$$
u_{j} \in V\left(s^{j}\right)-\bigcup_{i \neq j} V\left(s^{i}\right), \quad j=1, \ldots, h .
$$

Now choose complex numbers $\lambda_{1}, \ldots, \lambda_{h}$ such that

$$
\sum_{j=1}^{h} A_{j}^{1} \lambda_{j}=1 \quad \text { and } \quad \sum_{j=1}^{h} A_{j}^{i} \lambda_{j}=0 \text { for } i=2, \ldots, h
$$

and let $v_{j}=\lambda_{j} u_{j}, j=1, \ldots, h$. For any $s \in H^{0}\left(X, L^{*}\right)$, there exist $\mu_{i}$ such that $s=\sum_{i} \mu_{i} s^{i}$. In this case, $T s=\mu_{1}$. On the other hand,

$$
\Sigma^{h} s\left(v_{1}, \ldots, v_{h}\right)=\sum_{i=1}^{h} \sum_{j=1}^{h} \mu_{i} s^{i}\left(v_{j}\right)=\sum_{i=1}^{h} \sum_{j=1}^{h} \mu_{i} A_{j}^{i} \lambda_{j}=\sum_{i=1}^{h} \mu_{i} \delta^{1 i}=\mu_{1}
$$

Formula (12) completes the proof of the Lemma.

Proof of Proposition 3. Let $U$ be the subspace of $H^{0}\left(X, L^{*}\right)$ generated by the sections $s_{1}, \ldots, s_{N}$. Let $U^{\perp} \subset H^{0}\left(X, L^{*}\right)^{*}$ be the annihilator of $U$. Suppose $T \in U^{\perp}$. Then by Lemma 5 there exist vectors $v_{1}, \ldots, v_{h} \in L$ such that $T s=\Sigma^{h} s\left(v_{1}, \ldots, v_{h}\right)$. Now, since $T s_{i}=0$ for $i=1, \ldots, N$, the hypothesis on $g$ and the expression $T s=$ $\Sigma^{h} s\left(v_{1}, \ldots, v_{h}\right)$ imply that $T g=0$. Thus $g \in\left(U^{\perp}\right)^{\perp}=U$, as desired.

It is possible to prove Proposition 3 using the ideas in Theorem 1, but we feel that Proposition 3 is interesting in its own right. The operation $\Sigma^{k}$ can be thought of as a discrete version of the $(k-1)$-jet of a section. In fact, the $\Sigma^{k}$ contain more information than jets do, because one may look at points not on the diagonal. From this point of view the hypothesis of Proposition 3 says that the global section $g$ vanishes to order $h$ whenever the global sections $s_{1}, \ldots, s_{N}$ do.

In both Theorem 1 and Proposition 3 we conclude that a function $g$ must be a linear combination of some given functions $f_{j}$ (or $s_{j}$ ) under certain conditions. In each case these conditions guarantee that the function $\|f\|^{2}-|g|^{2}$ lies in $\mathcal{P}_{\infty}$. Thus Proposition 3 is an algebraic analogue of Lemma 2.

Let $S$ denote the set of singular globalizable metrics on $L$. Proposition 3 and Corollary 3 combine to show that $I(S) \leq \operatorname{dim} H^{0}\left(X, L^{*}\right)$. The ideas from Theorem 1 provide a stronger result on the stability index.

Theorem 2. (Stability of Positivity Conditions) Let $X$ be a compact complex manifold, and let $L$ be a holomorphic line bundle over $X$. Let $S$ be the set of globalizable metrics on $L$. Then $S$ is stable. In fact $I(S)$ is at most $\operatorname{dim}\left(H^{0}\left(X, L^{*}\right)\right)-1$.

Proof. We first observe that bounded meromorphic functions on $L$ must be constant. We can therefore virtually repeat the proof of Theorem 1 . Put $k=$ $\operatorname{dim}\left(H^{0}\left(X, L^{*}\right)\right)-1$. Let $R$ denote a globalizable metric, whose underlying Hermitian form is $C$. Note that $C$ has at most $k+1$ eigenvalues. Suppose that $R \in \mathcal{P}_{k}$; by the argument in Theorem 1, either $C$ has no negative eigenvalues, or at least $k+1$ positive eigenvalues. In the second case the total number of positive eigenvalues must 
equal $k+1$. Thus, in either case, $R$ is in $\mathcal{P}_{\infty}$. Therefore $S \cap \mathcal{P}_{k}=S \cap \mathcal{P}_{\infty}$, and hence $S$ is stable and $I(S)=k$.

4. Perturbations of the standard metric on $\mathbf{U}^{2 m}$. In this section we will show that the sets $\mathcal{P}_{k}(M)$ are distinct even when $M=\mathbf{C}^{2}$. We may also regard formula (13) below as arising from a family of globalizable metrics on the line bundle $\mathbf{U}^{2 m}$ over $\mathbf{P}_{1}$.

We consider a family of bihomogeneous polynomials defined on $\mathbf{C}^{2}$. These polynomials may be considered as perturbations of the natural example (on the diagonal) $z \rightarrow\|z\|^{4 m}=R_{0}(z, \bar{z})$. For each real number $\lambda$, and each positive integer $m$ we define

$$
R_{\lambda}(z, \bar{w})=\langle z, w\rangle^{2 m}-\lambda\left(z_{1} z_{2} \bar{w}_{1} \bar{w}_{2}\right)^{m}
$$

Note that $R_{\lambda}$ depends on both $\lambda$ and $m$. Let us write $S_{\lambda, m}$ for the set of polynomials of the form (13). Theorem 1 below provides precise information on when $R_{\lambda} \in \mathcal{P}_{k}$. We determine the set of values of $\lambda$ (in terms of $m$ ) for this inclusion to hold in the special cases $k=1, k=2$, and $k=\infty$. We also show that $\mathcal{P}_{k}=\mathcal{P}_{j}$ for $k \geq j \geq m+1$. Thus, $R_{\lambda} \in \mathcal{P}_{\infty}$ if and only if $R_{\lambda} \in \mathcal{P}_{k}$ whenever $k \geq m+1$. In particular $I\left(S_{\lambda, m}\right)=m+1$. Finally we determine the precise value of $\lambda$ for $R_{\lambda}$ to be in $\mathcal{P}_{m}$.

These results demonstrate an important fact about our positivity conditions.

Consequence. For each $m$, we have $\mathcal{P}_{m}\left(\mathbf{C}^{2}\right) \neq \mathcal{P}_{m+1}\left(\mathbf{C}^{2}\right)$. Thus $\mathcal{P}_{1}(M)$ is not stable already for $M=\mathbf{C}^{2}$.

Theorem 3. Fix $m$, and let $R_{\lambda}$ be defined by (13). The following statements hold:

1) $R_{\lambda} \in \mathcal{P}_{1}$ if and only if $\lambda \leq 2^{2 m}$.

2) $R_{\lambda} \in \mathcal{P}_{2}$ if and only if $\lambda \leq 2^{2 m-1}$.

3) $R_{\lambda} \in \mathcal{P}_{\infty}$ if and only if $\lambda \leq\left(\begin{array}{c}2 m \\ m\end{array}\right)$.

4) For each integer $k>m, R_{\lambda} \in \mathcal{P}_{k}$ if and only if $R_{\lambda} \in \mathcal{P}_{\infty}$.

5) $R_{\lambda} \in \mathcal{P}_{m}$ only if $\lambda \leq\left(\begin{array}{c}2 m \\ m\end{array}\right)+2$.

6) $I\left(S_{\lambda, m}\right)=m+1$.

Proofs. 1) To decide whether $\mathcal{P}_{1}$ holds is elementary. By homogeneity it suffices to assume that $\|z\|^{2}=1$; we then want the largest $\lambda$ such that

$$
1-\lambda\left|z_{1}\right|^{2 m}\left|z_{2}\right|^{2 m} \geq 0
$$

given that $\left|z_{1}\right|^{2}+\left|z_{2}\right|^{2}=1$. It is evident that the maximum value of $\left|z_{1}\right|^{2 m}\left|z_{2}\right|^{2 m}$ on the sphere occurs when $\left|z_{1}\right|^{2}=\left|z_{2}\right|^{2}=\frac{1}{2}$. Combining this observation with (14) shows that

$$
1-\frac{\lambda}{2^{2 m}} \geq 0
$$

which gives the result we want.

3) To decide when $\mathcal{P}_{\infty}$ holds is also elementary. On the diagonal we have

$$
R_{\lambda}(z, \bar{z})=\left(\left|z_{1}\right|^{2}+\left|z_{2}\right|^{2}\right)^{2 m}-\lambda\left(\left|z_{1}\right|^{2}\left|z_{2}\right|^{2}\right)^{m} .
$$

Expanding the left-hand side by the binomial theorem reveals that we obtain a squared norm if and only $\lambda$ is at most the coefficient there of $\left(\left|z_{1}\right|^{2}\left|z_{2}\right|^{2}\right)^{m}$; this coefficient is $\left(\begin{array}{c}2 m \\ m\end{array}\right)$. 
2) We next verify that $\mathcal{P}_{2}$ fails for $R_{\lambda}$ when $\lambda>2^{2 m-1}$. We choose the points $z=(1,1)$ and $w=(1,-1)$ and compute

$$
R_{\lambda}(z, \bar{z}) R_{\lambda}(w, \bar{w})-\left|R_{\lambda}(z, \bar{w})\right|^{2}
$$

there. We suppose that the expression in (15) is nonnegative. Using the orthogonality of $z$ and $w$, the computation simplifies and we obtain the condition

$$
0 \leq 2^{2 m}-2 \lambda
$$

which shows that the inequality fails for $\lambda>2^{2 m-1}$. To verify that $\mathcal{P}_{2}$ holds for $\lambda \leq$ $2^{2 m-1}$ one must first show that the cut-off value of $\lambda$ is determined when $\langle z, w\rangle=0$. We omit the considerable details; they are similar to the proof of 5). After verifying this fact, we must maximize $\left|z_{1} z_{2}\right|^{2 m}+\left|w_{1} w_{2}\right|^{2 m}$ given that $\left|z_{1}\right|^{2}+\left|z_{2}\right|^{2}=\left|w_{1}\right|^{2}+$ $\left|w_{2}\right|^{2}=1$ and that $z$ and $w$ are orthogonal. The maximum occurs at several points, including $\frac{1}{\sqrt{2}}(1,1)$ and $\frac{1}{\sqrt{2}}(1,-1)$. These points yield the desired statement about $\lambda$.

We prove 4) in Proposition 4 below. We prove 5) in Proposition 5 below. Statement 6) follows by combining statements 4) and 5).

Before proving these statements we interpret them when $m=1$ and $m=2$. First we set $m=1$ and recover information implied by Theorems 1 and 2 :

$$
\mathcal{P}_{1} \cap S_{\lambda, 1} \neq \mathcal{P}_{2} \cap S_{\lambda, 1}
$$

but that

$$
\mathcal{P}_{2} \cap S_{\lambda, 1}=\mathcal{P}_{\infty} \cap S_{\lambda, 1}
$$

Thus $I\left(S_{\lambda, 1}\right)=2$.

Next consider $m=2$; we see that

$$
\mathcal{P}_{1} \cap S_{\lambda, 2} \neq \mathcal{P}_{2} \cap S_{\lambda, 2} \neq \mathcal{P}_{3} \cap S_{\lambda, 2}
$$

but that

$$
\mathcal{P}_{3} \cap S_{\lambda, 2}=\mathcal{P}_{\infty} \cap S_{\lambda, 2} .
$$

Thus $I\left(S_{\lambda, 2}\right)=3$. This information does not follow from Theorems 1 and 2 .

It is instructive to prove 4) of Theorem 3 first when $m=2$. By Corollary 3 we must determine the set of $\lambda$ for which the inequality

$$
\begin{gathered}
\left|z_{1}^{4}+w_{1}^{4}+u_{1}^{4}\right|^{2}+4\left|z_{1}^{3} z_{2}+w_{1}^{3} w_{2}+u_{1}^{3} u_{2}\right|^{2}+4\left|z_{1} z_{2}^{3}+w_{1} w_{2}^{3}+u_{1} u_{2}^{3}\right|^{2}+\left|z_{2}^{4}+w_{2}^{4}+u_{2}^{4}\right|^{2} \\
\geq(\lambda-6)\left|\left(z_{1} z_{2}\right)^{2}+\left(w_{1} w_{2}\right)^{2}+\left(u_{1} u_{2}\right)^{2}\right|^{2}
\end{gathered}
$$

holds for all triples of points $z, w, u$.

(16) is trivial for $\lambda \leq 6$; of course 6 is the cut-off point for being a squared norm. We use Corollary 4. Suppose that (16) holds, and we can show that the left-hand side of (16) can vanish when the right-hand side does not vanish. We see that the inequality will fail for any $\lambda$ larger than 6 .

Let $h$ denote the polynomial in six variables given by $\left(z_{1} z_{2}\right)^{2}+\left(w_{1} w_{2}\right)^{2}+\left(u_{1} u_{2}\right)^{2}$. Let $J$ denote the ideal in the polynomial ring given by

$$
J=\left(z_{1}^{4}+w_{1}^{4}+u_{1}^{4}, z_{1}^{3} z_{2}+w_{1}^{3} w_{2}+u_{1}^{3} u_{2}, z_{1} z_{2}^{3}+w_{1} w_{2}^{3}+u_{1} u_{2}^{3}, z_{2}^{4}+w_{2}^{4}+u_{2}^{4}\right) .
$$


To verify the existence of points as in the previous paragraph, it suffices to prove that $h$ is not in the radical of $J$. Dan Lichtblau of Wolfram Research verified this and other statements for us using the Groebner basis algorithm from Mathematica. After knowing this information, it is natural to instead seek points that work.

First we choose $z_{1} z_{2}=w_{1} w_{2}=u_{1} u_{2}=1$. With these choices $h$ will have the value 3. Next we assume that $\left|z_{1}\right|^{2}=\left|w_{1}\right|^{2}=\left|u_{1}\right|^{2}=1$. By doing so, the four equations defined by the vanishing of the generators of $J$ become the two equations (17) and (18) and their complex conjugates.

$$
\begin{aligned}
& z_{1}^{4}+w_{1}^{4}+u_{1}^{4}=0 \\
& z_{1}^{2}+w_{1}^{2}+u_{1}^{2}=0
\end{aligned}
$$

We choose the three values to equal $1, b$, and $b^{2}$, where $b$ is a primitive third root of unity. Then both (17) and (18) become $1+b+b^{2}=0$, which holds since $b^{3}=1$ and $b \neq 1$. This verifies statement 4 ) of Theorem 3 when $m=2$.

We now generalize this proof.

Proposition 4. (Stability) For each positive integer $m$, and each real $\lambda$, let $S_{\lambda, m}$ denote the set of Hermitian symmetric functions $R_{\lambda}$ on $\mathbf{C}^{2}$ satisfying (19):

$$
R_{\lambda}(z, \bar{w})=\langle z, w\rangle^{2 m}-\lambda\left(z_{1} z_{2} \bar{w}_{1} \bar{w}_{2}\right)^{m} .
$$

Then, for $k \geq m+1$, the sets $S_{m, \lambda} \cap \mathcal{P}_{k}$ are all the same. In particular, $R_{\lambda} \in \mathcal{P}_{k}$ if and only if $\lambda \leq\left(\begin{array}{c}2 m \\ m\end{array}\right)$, and $I\left(S_{\lambda, m}\right) \leq m+1$.

Proof. We mimic the proof in the special case $m=2$. Let $g(z)=z_{1}^{m} z_{2}^{m}$. We observe that, for $z_{1} \neq 0$, we have $g\left(z_{1}, \frac{1}{z_{1}}\right)=1$. For any collection of points $W_{j}$ in $\mathbf{C}^{2}$ of the form $\left(w_{j}, \frac{1}{w_{j}}\right)$ we therefore have $\Sigma^{N} g\left(W_{1}, \ldots, W_{N}\right)=N$.

Let $J$ denote the ideal analogous to the ideal in the special case above. The generators of $J$ are the functions $\Sigma^{N} h_{j}$, where $h_{j}(z)=z_{1}^{2 m-j} z_{2}^{j}$, for $0 \leq j<m$ or $m+1 \leq j \leq 2 m$. We claim that, for $N=m+1$, we can find $N$ points $W_{i}$ in $\mathbf{C}^{2}$ of the form $W_{i}=\left(w_{i}, \frac{1}{w_{i}}\right)$ such that $\Sigma^{N} f\left(W_{1}, \ldots, W_{N}\right)=0$. By Corollary 4 this implies the desired result for $R_{\lambda}$.

We will find solutions where $\left|w_{i}\right|^{2}=1$. Assume this condition. Then the $2 \mathrm{~m}$ equations $h_{j}=0$ become $m$ equations and their conjugates. It therefore suffices to verify the first $m$ equations:

$$
\sum_{i=1}^{m} w_{i}^{2 m-2 j}=0
$$

for $0 \leq j \leq m-1$. To satisfy the equations in $(20)$ we set $\left(w_{1}\right)^{2}=1,\left(w_{2}\right)^{2}=\eta$, $\left(w_{3}\right)^{2}=\eta^{2}$, and so on, where $\eta$ is a primitive $m+1$-st root of unity. We obtain the $m$ equations

$$
\sum_{i=1}^{m} \eta^{(i-1)(m-j)}=\sum_{i=0}^{m-1}\left(\eta^{m-j}\right)^{i}=0 .
$$

For each $j$, equation (21) holds because $\eta^{m-j}$ is an $m+1$-st root of unity but does not equal 1.

Proposition 5. Statement 5) from Theorem 3 holds. 
Proof. We have $R_{\lambda}=\|f\|^{2}-\lambda\|g\|^{2}$ on the diagonal, where $f$ and $g$ are as follows:

$$
\begin{gathered}
\|f(z)\|^{2}=\|z\|^{4 m}-\left(\begin{array}{c}
2 m \\
m
\end{array}\right)\left|\left(z_{1} z_{2}\right)^{m}\right|^{2} \\
\|g(z)\|^{2}=\left|\left(z_{1} z_{2}\right)^{m}\right|^{2} .
\end{gathered}
$$

Note that the right-hand side of (22) is a squared norm. The components of $f$ are all the homogeneous polynomials of degree $2 m$ except for the middle term $\left(z_{1} z_{2}\right)^{m}$, and the coefficients are the binomial coefficients.

By Corollary 3 the largest $\lambda$ for which $\mathcal{P}_{k}$ holds is given by the infimum of the expression

$$
\frac{\left\|\Sigma^{k} f\left(w_{1}, \ldots, w_{k}\right)\right\|^{2}}{\left\|\Sigma^{k} g\left(w_{1}, \ldots, w_{k}\right)\right\|^{2}} .
$$

In (24), $w_{j}=\left(\left(w_{j}\right)_{1},\left(w_{j}\right)_{2}\right)$. Let $k=m$. Since $(24)$ is homogeneous of degree 0 , and because of the symmetry, the infimum happens where $w_{k}=\left(1, \eta^{k-1}\right)$ and $\eta^{m}=1$. Plugging this into (24), and using properties of roots of unity, all the terms in $\left\|\Sigma^{m} f\right\|^{2}$ vanish except the terms $\left|\Sigma\left(\left(w_{j}\right)_{1}\right)^{m}\right|^{2}$ and $\left|\Sigma\left(\left(w_{j}\right)_{2}\right)^{m}\right|^{2}$. These each give the value $m^{2}$. On the other hand, the value of $\Sigma^{m} g$ at these points is $m$. Hence the value of (24) at these points is 2 . Incorporating the terms $\left(\begin{array}{c}2 m \\ m\end{array}\right)\left|\left(z_{1} z_{2}\right)^{m}\right|^{2}$ gives the desired result for $R_{\lambda}$, where $f$ and $g$ are expressed in the forms (22) and (23).

This calculation proves that $R_{\lambda}$ is not in $\mathcal{P}_{m}$ when $\lambda>\left(\begin{array}{c}2 m \\ m\end{array}\right)+2$. Containment does hold for this value, because the infimum in (24) is attained at these points. Even though the numerator vanishes on a two-dimensional variety, the denominator vanishes there as well, and the limiting value of the ratio, as we approach this variety, exceeds 2 .

REMARK 2. For any homogeneous polynomial mapping $f$ of degree $m$ in two variables, the variety $V\left(\Sigma^{k} f\right)$ is positive dimensional for $k \geq 2$. On the other hand, for the functions in Theorem 3, we have $V\left(\Sigma^{k} f\right) \subset V\left(\Sigma^{k} g\right)$ for $k \geq 2$. The key point is that the infimum of the ratio happens away from the variety $V\left(\Sigma^{k} f\right)$.

5. The role of $\mathcal{P}_{2}$. The main purpose of this section is to discuss $\mathcal{P}_{2}$ in more detail. For completeness we first recall and augment our list of geometric positivity conditions. Suppose that $R=R_{C}$ is a globalizable metric as in (6). The following positivity conditions all arise:

Definition 5. (Geometric positivity conditions)

1) $\mathcal{G} \mathcal{P}_{\infty}$ : The matrix $\left(c_{\alpha \beta}\right)$ of coefficients is nonnegative definite.

2) $\mathcal{G} \mathcal{P}_{\infty}^{\sharp}$ : The matrix $\left(c_{\alpha \beta}\right)$ of coefficients is positive definite.

3) $\mathcal{G}$ : There is an integer $d$ such that

$$
R^{d}(z, w)=\sum_{\mu, \nu} E_{\mu \nu} \psi_{\mu} \bar{\psi}_{\nu}
$$

where the matrix $\left(E_{\mu \nu}\right)$ is nonnegative definite.

4) $\mathcal{G}^{\sharp}$ : There is an integer $d$ such that

$$
R^{d}(z, w)=\sum_{\mu, \nu} E_{\mu \nu} \psi_{\mu} \bar{\psi}_{\nu}
$$


where the matrix $\left(E_{\mu \nu}\right)$ is positive definite.

5) $R \in \mathcal{P}_{2}$; equivalently the global Cauchy-Schwarz inequality holds:

$$
|R(z, \bar{w})|^{2} \leq R(z, \bar{z}) R(w, \bar{w}) .
$$

6) A sharp form of the global Cauchy-Schwarz inequality holds:

$$
|R(z, \bar{w})|^{2} \leq R(z, \bar{z}) R(w, \bar{w}),
$$

and equality happens only in some specified precise setting.

7) The function $z \rightarrow \log R(z, \bar{z})$ is plurisubharmonic.

8) The function $z \rightarrow R(z, \bar{z})$ is plurisubharmonic.

Items 2), 4) and 6) are sharp forms of items 1), 3), and 5). We could of course also introduce sharp forms of 7) and 8).

By elementary linear algebra 1 ) is equivalent to the existence of a (finite-dimensional) Hilbert space valued mapping $f$ representing $R$ and is thus is the same as $\mathcal{P}_{\infty}$. One can easily show [D4] that 3) implies 5). By definition, 5) is the same as $R \in \mathcal{P}_{2}$. The assumption that $R$ is a (possibly degenerate) metric is of course the same as $R \in \mathcal{P}_{1}$. Conditions 7) and 8) lie between $\mathcal{P}_{1}$ and $\mathcal{P}_{2}$; It is shown in [D4] that 5) implies 7 ) implies 8 ). We revisit our main example below to show that each converse assertion fails. Statements 7) and 8) are equivalent in the bihomogeneous case; see [D3].

Theorem 3 Revisited. Let $M$ be $\mathbf{C}^{2}$. For each real number $\lambda$ we define a Hermitian symmetric polynomial $r_{\lambda}$ by

$$
r_{\lambda}(z, \bar{z})=\left(\left|z_{1}\right|^{2}+\left|z_{2}\right|^{2}\right)^{4}-\lambda\left|z_{1} z_{2}\right|^{4} .
$$

(Thus $m=2$ in Theorem 3.) The following statements are true:

2.1) $r_{\lambda} \in \mathcal{P}_{1}$ if and only if $\lambda \leq 16$. (This is the condition for being a metric.)

2.2) $r_{\lambda}$ is plurisubharmonic (on the diagonal) for $\lambda \leq 12$.

2.3) $\log \left(r_{\lambda}\right)$ is plurisubharmonic (on the diagonal) for $\lambda \leq 12$.

2.4) $r_{\lambda} \in \mathcal{P}_{2}$ if and only $\lambda \leq 8$.

2.5) For $k \geq 3, r_{\lambda} \in \mathcal{P}_{k}$ if and only if $r_{\lambda} \in \mathcal{P}_{\infty}$; this condition occurs if and only if $\lambda \leq 6$.

REMARK 3. Theorem 3 therefore reveals that four of the five conditions are distinct already for bihomogeneous polynomials of degree 8 in $\mathbf{C}^{2}$. If we set $z_{2}=1$, and consider the resulting function of one variable, then the condition $\lambda \leq 12$ for logarithmic plurisubharmonicity is unchanged; the condition for plurisubharmonicity becomes $\lambda \leq \frac{3}{32}(69+11 \sqrt{33})$. (See [D4]). The value of this expression is approximately 12.39. Thus all five conditions are distinct for polynomials in two variables.

Condition 7) from Definition 5 is equivalent to the strict negativity of the bundle $L$ using the metric $R$. The Cauchy-Schwarz inequality on a globalizable metric is thus an intermediate condition between being a holomorphic pullback and having negative curvature. We therefore give a different characterization of $\mathcal{P}_{2}$; this result holds for Hermitian symmetric functions not necessarily arising from metrics.

In order to state the result in a geometric fashion we introduce two pieces of notation. Suppose that $f, g: M \rightarrow \mathcal{H}$ are holomorphic mappings. We write $f \wedge g$ for the skew-symmetric function from $M \times M$ to $\mathcal{H} \otimes \mathcal{H}$ defined by 


$$
(f \wedge g)(z, w)=f(z) \otimes g(w)-f(w) \otimes g(z)
$$

We also write $\theta_{f}(z, w)$ for the angle between the vectors $f(z)$ and $f(w)$ in $\mathcal{H}$. Finally we note the standard identity

$$
\|f(z) \otimes f(w)\|^{2}=\|f(z)\|^{2}\|f(w)\|^{2}=\left.\|f(z)\|\right|^{2}\|f(w)\|^{2} \sin ^{2} \theta_{f}(z, w)+|\langle f(z), f(w)\rangle|^{2} .
$$

Let $(f, g): M \rightarrow \mathcal{H} \times \mathcal{H}$ be a holomorphic representation of $R$. We have the following beautiful geometric interpretation of $\mathcal{P}_{2}$. When $k=2$ the formulas in Lemma 6 provide an alternative way of expressing the determinant (3) used earlier.

LEMma 6 . Let $R=\|f\|^{2}-\|g\|^{2} \geq 0$ on the diagonal; then $R \in \mathcal{P}_{2}$ if and only if

$$
\|(f \wedge g)(z, w)\|^{2} \leq\|f(z)\|^{2}\|f(w)\|^{2} \sin ^{2} \theta_{f}(z, w)+\left.\|g(z)\|\right|^{2}\|g(w)\|^{2} \sin ^{2} \theta_{g}(z, w)
$$

for all $z$ and $w$ in $M$. This inequality can also be written as

$\left\|(f \wedge g)(z, w)||^{2}+|\langle f(z), f(w)\rangle|^{2}+|\langle g(z), g(w)\rangle|^{2} \leq\right\| f(z)\left\|\left.\right|^{2}\right\| f(w)\left\|^{2}+\right\| g(z)\left\|\left.\right|^{2}\right\| g(w) \|^{2}$

Proof. We apply the definition of $\mathcal{P}_{2}$; since $R$ is nonnegative at each point, it is in $\mathcal{P}_{2}$ if and only if the determinant $\Delta_{R}^{2}$ is nonnegative at each pair of points $z$ and $w$. The determinant information yields

$$
|R(z, \bar{w})|^{2} \leq R(z, \bar{z}) R(w, \bar{w})
$$

Next replace $R(z, \bar{w})$ by its holomorphic representation in (30). Finally expand, collect terms, and use (27). It follows that inequality (28) is equivalent to (30). It is clear that (28) and (29) are also equivalent.

Inequalities (28) and (29) differ from the usual Cauchy-Schwarz inequality because of the presence of the term $\|(f \wedge g)(z, w)\|^{2}$, which relates $f$ and $g$. Inequalities (29) and (30) are equivalent forms of the nonnegativity of $\Delta_{R}^{2}$ as in (3).

As in the proof of Theorem 1, Lemma 6 is especially useful when $g$ is scalar-valued. In that case $\sin ^{2} \theta_{g}(z, w)$ vanishes, and formulas (28) and (29) simplify.

\section{REFERENCES}

[A] Aronszajn, N., Theory of Reproducing Kernels, Trans. A. M. S., 68 (1950), pp. 337-404.

[AM] Agler, Jim, and McCarthy, John E., Pick Interpolation and Hilbert Function Spaces, Graduate Studies in Mathematics, Volume 44, American Mathematical Society, Providence, Rhode Island, 2000.

[CD1] Catlin, David W. and D'Angelo, John P., Positivity conditions for bihomogeneous polynomials, Math Research Letters, 4 (1997), pp. 1-13.

[CD2] Catlin, David W. and D'Angelo, John P., An isometric imbedding theorem for holomorphic bundles, Math Research Letters, 6 (1999), pp. 1-18.

[CW] Cole, Brian J., and Wermer, John, Ando's Theorem and Sums of Squares, Indiana Univ. Math J., 48 (1999), pp. 767-791.

[D1] D'Angelo, John P., Positivity conditions and squared norms of holomorphic polynomial mappings, Contemporary Mathematics, 251 (2000), pp. 163-172. 
[D2] D'Angelo, John P., Positivity conditions for real-analytic functions, pp. 103-123 in Complex Analysis and Geometry, edited by Jeffery D. McNeal, Ohio State Univ. Math Res. Inst. Publ 9, Walter de Gruyter, Berlin, 2001.

[D3] D'Angelo, John P., Bordered complex Hessians, Journal of Geometric Analysis, 11:4 (2001), pp. $559-569$

[D4] D'Angelo, John P., Inequalities from Complex Analysis, Carus Mathematical Monograph No. 28, Mathematics Association of America, 2002.

[Siu] Siu, Y. T., Invariance of Plurigenera, Invent. Math., 134:3 (1998), pp. 661-673.

[S] Stewart, J., Positive definite functions and generalizations: an historical survey, Rocky Mountain J. Math., 6 (1976), pp. 409-434. 
